\title{
Idiopathic nonspecific interstitial pneumonia: an interstitial lung disease associated with autoimmune disorders?
}

\author{
M. Romagnoli*, C. Nannini ${ }^{\#}$, S. Piciucchi ${ }^{\mp}$, F. Girelli ${ }^{+}$, C. Gurioli*, G. Casoni*, \\ C. Ravaglia*, S. Tomassetti*, Ch. Gurioli*, G. Gavelli ${ }^{\dagger}$, A. Carloni $^{\S}$, A. Dubini $^{f}$, \\ F. Cantini ${ }^{\#}$, M. Chilosi** and V. Poletti*
}

ABSTRACT: Recent evidence suggests that idiopathic nonspecific interstitial pneumonia (iNSIP) is a distinct clinical entity amongst other idiopathic interstitial pneumonias, and some data seem to suggest a possible pathogenetic role of autoimmunity. The aim of the present study was to assess if iNSIP might represent an early lung manifestation of an autoimmune disease.

After initial review of cases found in the medical records database by searching for the term "NSIP" ( $n=63), 37$ iNSIP cases were identified, and were re-evaluated using a dynamic integrated multidisciplinary approach.

27 cases with iNSIP were selected for the study. Mean \pm SD age at first respiratory symptom was $54.2 \pm 8 \mathrm{yrs}, 70 \%$ were females, and $59 \%$ were never-smokers. At follow-up (mean \pm SD $59.7 \pm 29$ months, range 12-138 months), autoimmune diseases occurred in 14 (52\%) patients, with seven $(26 \%)$ cases of autoimmune thyroiditis, six (22\%) of undifferentiated connective tissue disease and three (11\%) of connective tissue disease. Patients developing autoimmune diseases were older and more frequently never-smoking females.

In $>\mathbf{5 0} \%$ of patients diagnosed with iNSIP, evidence of autoimmune diseases develops within 2 yrs, suggesting a probable link between the clinical entity of iNSIP and autoimmune disorders.

KEYWORDS: Autoimmune disease, connective tissue disease, idiopathic interstitial pneumonia, lung, thyroiditis

$\mathbf{N}$ onspecific interstitial pneumonia (NSIP) was initially defined as a histopathological pattern [1] that can be found in the presence of a wide variety of clinical and radiological entities [2]. Conventionally, and based on clinical grounds, NSIP is aetiologically defined as "idiopathic" [3] or "secondary", depending on the absence or presence of known aetiological conditions for NSIP, with the recommendation that the "clinical diagnosis" of NSIP should be reserved only for idiopathic and biopsy-proven cases when no causative factor can be identified [2, 4].

Interestingly, the recent report of an American Thoracic Society project has suggested that idiopathic (i)NSIP does represent a distinct clinical entity, notably occurring most frequently in middle-aged and never-smoking females showing a good long-term prognosis [5]. The histopathological diagnosis in the diagnostic approach of iNSIP is still based on surgical lung biopsy [3], although in some cases the need for histological proof has been questioned [6].

Two recent studies have revealed a probable link between iNSIP and autoimmune diseases [7, 8]. In a US study, it was found that $>80 \%$ of 28 patients diagnosed with iNSIP presented with an undifferentiated connective tissue disease (UCTD) from the beginning [7] and, in an Asian cohort of iNSIP, connective tissue diseases (CTDs) occurred during follow-up in $10 \%$ of cases [8], suggesting a possible role of autoimmunity in iNSIP.

Other pathogenetic mechanisms have been proposed in iNSIP. The association of the presence of emphysema in smoking patients with iNSIP [9], and the evolution into a pattern resembling fibrosing NSIP of some cases of desquamative interstitial pneumonia, typically associated with cigarette smoking [10], provide indirect support also for a smoking-related pathogenesis, at least in some iNSIP cases.
AFFILIATIONS

Depts of *Pulmonology,

+Internal Medicine-Rheumatology,

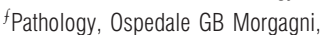
Forlì,

\#Dept of Rheumatology, Ospedale

Generale, Prato,

"Dept of Radiology, Istituto

Scientifico Romagnolo per lo Studio

e la Cura dei Tumouri, Meldola-Forli,

${ }^{\S}$ Dept of Radiology, Ospedale

Generale, Terni, and

${ }^{*}$ Dept of Histopathology, University

of Verona, Verona, Italy.

CORRESPONDENCE

V. Poletti

Pulmonology Unit, Dept of Thoracic Diseases

Pierantoni-Morgagni Hospital

Via C. Forlanini

34

47100 Forlì

Italy

E-mail: v.poletti@ausl.fo.it

Received:

June 202010

Accepted after revision:

Jan 072011

First published online:

Jan 272011 
We hypothesised that the distinct clinical entity iNSIP might be associated with an autoimmune background that later reveals itself as an organ-specific or a systemic autoimmune disease. To this end, the evaluation of the occurrence of autoimmune diseases in previously established and surgically proven iNSIP was conducted.

\section{METHODS}

\section{Patients and inclusion criteria}

The initial point for the enrolment of patients was a pathological diagnosis of NSIP based on a surgical lung biopsy, as previously described [3]. These patients were identified in the medical record database of our dept by searching between January 1, 1998 and July 2009 for the term "NSIP". After initial review of all identified NSIP cases, only those with an iNSIP and complete data for autoimmunity assessment (both serological and clinical data) were included in the study. The included cases were then discussed, reviewed at a multidisciplinary conference and the diagnosis of iNSIP was finally based on a dynamic integrated multidisciplinary approach, as previously described [5].

The diagnosis of the various CTD entities was made according to previously published criteria [11-14], including mixed CTD [15] and UCTD (evidence of systemic inflammation in the absence of infection and symptoms associated with CTD, not fulfilling the classification criteria for defined CTD) [7, 16, 17]. Briefly, patients were asked about the presence of Raynaud's phenomenon, arthralgia/multiple joint swelling, photosensitivity, unintentional weight loss, morning stiffness, dry mouth or dry eyes (sicca features), dysphagia, recurrent unexplained fever, gastro-oesophageal reflux, skin changes (rash), oral ulceration, nonandrogenic alopecia, and proximal muscle weakness. Diagnosis of autoimmune thyroiditis was based on the presence of anti-thyroid antibodies [18] and a diffuse thyroid hypoechogenicity at ultrasonography [19].

All cases with established clinical, laboratory, radiological or pathological criteria suggestive for interstitial lung disease (ILD) other than iNSIP, or cases with a documented drug, airborne antigen and occupational exposure were excluded from the study. Cases that met, at the first respiratory symptom, rheumatological criteria for CTD, UCTD, mixed CTD and/or criteria for autoimmune thyroid disorders were also excluded from the analysis.

\section{Follow-up assessment}

Follow-up evaluation of the patients included in the study comprised the following once per year: 1) clinical and physical examination; 2) laboratory assessment (including autoimmune profile, e.g. rheumatoid factor, complement C3 and C4, antinuclear antigen, anti-extractable nuclear antigens, anti-citrulline autoantibodies, anti-thyroglobulin, anti-thyrotropin receptor and anti-thyroid peroxidase (anti-TPO) autoantibodies, erythrocyte sedimentation rate (ESR) and/or C-reactive protein (CRP) levels and hepatitis C and B virus serology); 3) pulmonary function tests; 4) high-resolution computed tomography (HRCT) of the chest; and 5) rheumatologist evaluation. When indicated by rheumatologists and/or endocrinologists because of thyroid disorders, further additional tests were performed (e.g. capillaroscopy, hands and feet bone radiography, Schirmer test, muscle and/or labial gland biopsy, electromyography, digestive tract radiography, and thyroid ultrasound).

\section{Pathological and radiological analysis}

All lung biopsy specimens were independently reviewed by two pulmonary pathologists, without knowledge of the clinical or radiological data. At least two different lobes of the lung were available for review in each patient. A consensus was reached for patients where there was initial disagreement.

HRCT images were reviewed in consensus by chest radiologists on a separate workstation and terminology was based on the Fleischner Society glossary [20].

As previously described [21], the lungs were divided into six zones (upper, middle and lower zones in both lungs) to quantify distribution of parenchymal changes, fibrosis was quantified by two scores (interstitial changes and traction bronchiectasis), and images were scored as grade 0 to grade 3 . The scores for the five regions were summed to provide an overall "coarseness score" (range 0-15) [21].

\section{Statistical analysis}

Continuous data are expressed as means \pm SD. Categorical data are expressed as percentages. Comparisons of clinical, functional and radiological parameters for each patient were made between the time of the first respiratory symptoms and the last follow-up evaluation. Comparisons between groups were made using an unpaired t-test, Chi-squared test, or Fisher's exact test as appropriate. Statistical significance was defined as a p-value of $<0.05$. All analysis was performed with StatView statistical software (version 5.0; SAS Institute Inc., Cary NC, USA).

\section{RESULTS}

\section{Demographic and clinical characteristics}

The analysis was conducted in the Pulmonology Unit of Forlì Hospital, Italy, a tertiary referral centre for patients with ILDs from all areas in Italy. After the initial review of charts, we identified 63 patients with a pathological diagnosis of NSIP. 37 $(58.7 \%)$ cases were considered to be iNSIP at the initial evaluation. Of the 37 NSIP cases classified as idiopathic, 10 patients were excluded from the analysis at the multidisciplinary evaluation (fig. 1).

The demographic and clinical characteristics of the 27 cases of iNSIP selected for the analysis are shown in table 1. Age (mean $\pm \mathrm{SD}$ ) at first respiratory symptom was $54.2 \pm 8$ yrs, and the majority of patients were female and never-smokers. All patients were Caucasians (from North, Central and South areas of Italy, and one patient from Lebanon). The mean follow-up (mean $\pm \mathrm{SD}$ ) was $59.7 \pm 29$ months, with a range of $12-$ 138 months. At first manifestation, most of patients presented with dyspnoea (85\% of patients), and cough in 33\%. One patient died (patient 7, table 2) after 18 months of follow-up because of a severe acute respiratory failure (mortality incidence $3.7 \%$ ) related to an acute exacerbation of NSIP.

All 27 patients were treated with tapering doses of systemic corticosteroids (prednisone $1 \mathrm{mg} \cdot \mathrm{kg}^{-1} \cdot \mathrm{day}^{-1}$ ) for at least $1 \mathrm{yr}$. In addition to prednisone, 12 out of the total 15 patients with "fibrosing NSIP", and the three patients with "fibrosing NSIP plus other patterns" also received cyclophosphamide $\left(100 \mathrm{mg} \cdot \mathrm{day}^{-1}\right)$ for at least $1 \mathrm{yr}$ (when tolerated). Of the nine patients with "cellular NSIP", in two cases, azathioprine and, in three cases, cyclophosphamide was added to prednisone for $1 \mathrm{yr}$. 


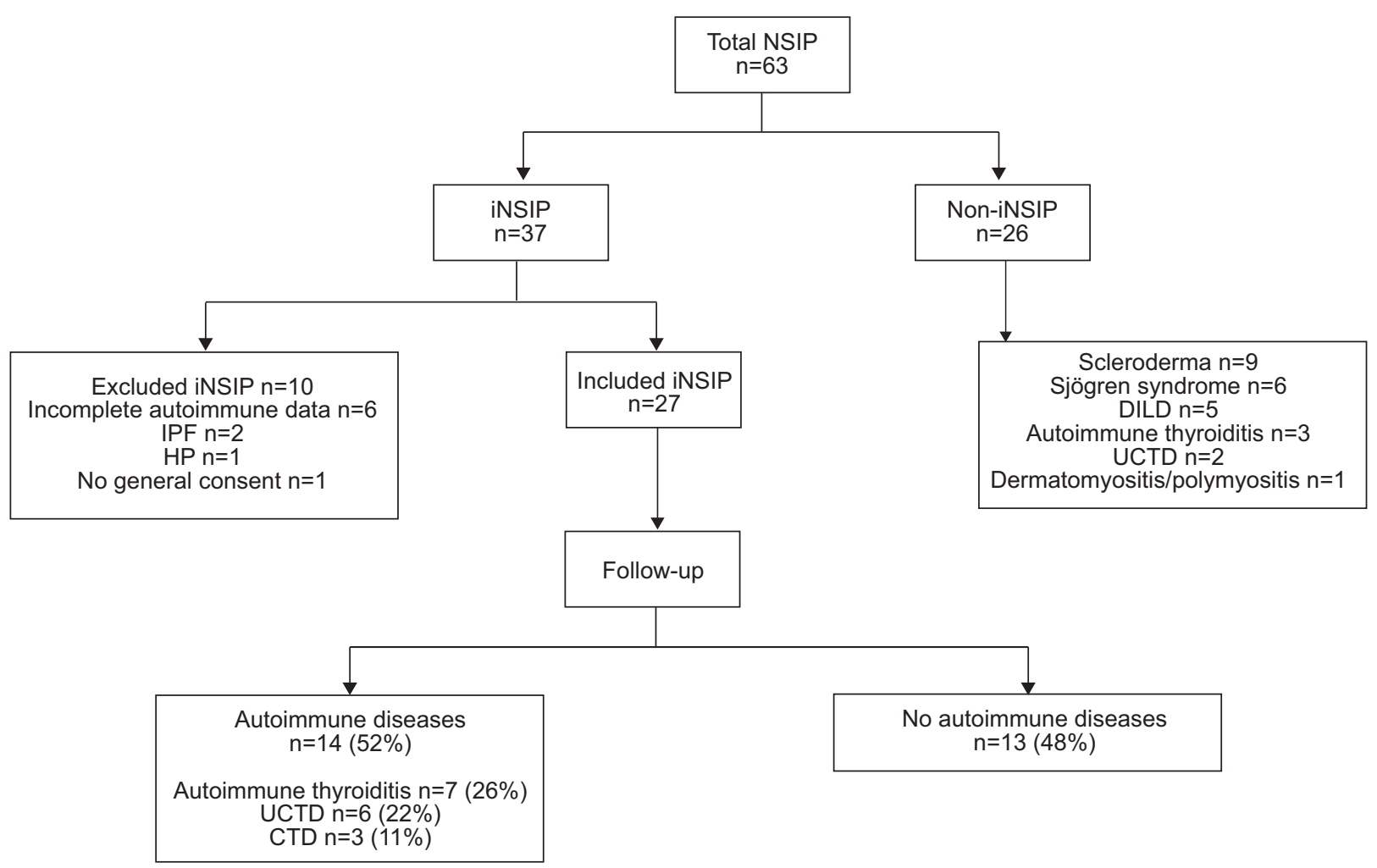

FIGURE 1. Patients flow chart. A total number of 37 idiopathic nonspecific interstitial pneumonia (iNSIP) cases were identified and 27 were finally included for analysis. At follow-up, 52\% of patients developed autoimmune diseases. CTD: connective tissue disease; DILD: drug-induced lung disease; UCTD: undifferentiated connective tissue disease; IPF: idiopathic pulmonary fibrosis; HP: hypersensitivity pneumonitis.

\section{Autoimmune findings}

Among the 27 patients with the diagnosis of iNSIP, a total of 14 $(52 \%)$ patients later developed an autoimmune disease (table 2). The mean $\pm S D$ (range) interval to an autoimmune disease manifestation was $22 \pm 18$ months (range 12-72 months) from the initial diagnosis of iNSIP. At the time of the present analysis, 15 patients had a follow-up of $<72$ months (eight of whom had not developed any autoimmune disease).

At the time of the first respiratory symptoms, four (14.8\%) patients presented with symptoms and signs suggestive of autoimmune disease in the absence of positive serological results, three $(11 \%)$ patients presented with positive serological results and seven patients showed increased ESR levels (26\%) in the complete absence of any symptom or sign for autoimmune disease (table 2).

At follow-up, autoimmune thyroiditis occurred in seven (26\%) patients, UCTD in six $(22 \%)$, and CTD in three $(11 \%)$ patients (fig. 1, table 2). In two cases, patients developed two different autoimmune diseases (patients 21 and 27; table 2): autoimmune thyroiditis in combination with rheumatoid arthritis and with a UCTD, respectively. The type of autoimmune thyroiditis was chronic Hashimoto's thyroiditis in all seven patients. In detail, we observed clinical hypothyroidism in four cases, with low free T4 levels and raised thyroid stimulating hormone (TSH) levels. In the remaining three cases, patients presented with subclinical hypothyroidism, with normal thyroid hormone levels and a rise in TSH. TPO and thyroglobulin antibodies both became positive in the follow-up of five out of the total seven patients, whereas in two patients, only one thyroid antibody became positive (table 2, patients 19 and 27; table 2). Thyroid ultrasound examination documented a diffuse hypoechoic pattern in all cases.

Two $(66 \%)$ out of the three patients presenting with positive serological results at onset (patients 2 and 20) developed an UCTD at follow-up. Of the eight patients with increased ESR levels at onset, an autoimmune disease occurred in seven $(87.5 \%)$ cases (OR 13; $\mathrm{p}=0.03)$ (table 2).

Of the four patients displaying systemic symptoms (and negative serology) at the time of first respiratory symptoms, three $(75 \%)$ developed autoimmune diseases at follow-up.

\section{Characteristics of patients with and without autoimmune diseases}

The characteristics of patients who developed autoimmune diseases after the initial diagnosis of iNSIP are shown in table 3. Patients who later developed autoimmune diseases at follow-up were more likely to be older and never-smoking females, as compared with patients who did not.

A tendency to have a less severe restrictive disorder, but more compromised DL,CO values, was observed in the group of patients who developed autoimmune diseases at follow-up. The analysis of the final HRCT scan score did not show any significant statistical difference between the two groups, 
TABLE 1 Patient demographic and clinical characteristics

Demographic or

clinical characteristic

\begin{tabular}{lc} 
Total patients $\mathbf{n}$ & 27 \\
Age at first respiratory symptom yrs (range) & $54.2 \pm 8(40-68)$ \\
Sex & $19(70)$ \\
Female & $8(30)$ \\
Male & \\
Smoking history & $16(59)$ \\
$\quad$ Never-smokers & $11(41)$ \\
Ex-smokers & $21 \pm 21(5-80)$ \\
Mean pack-yrs \pm SD (range) & \\
NSIP histopathology pattern & $15(56)$ \\
Fibrosing & $9(33)$ \\
Cellular & $3(11)$ \\
Fibrosing NSIP plus other patterns & \\
Follow-up months & $59.7 \pm 29(12-138)$ \\
Mean pack-yrs \pm SD (range) & \\
Respiratory symptoms & \\
Dyspnoea & $23(85)$ \\
$\quad$ At first respiratory symptoms & $19(70.3)$ \\
At follow-up & \\
Cough & $9(33.3)$ \\
$\quad$ At first respiratory symptoms & $7(26)$ \\
At follow-up & \\
FVC \% pred & $76 \pm 24$ \\
At first respiratory symptoms & $79 \pm 20$ \\
At follow-up & \\
FEV $1 \%$ pred & $81.3 \pm 19$ \\
At first respiratory symptoms & $80 \pm 22$ \\
At follow-up & \\
DL,Co & $57.7 \pm 14$ \\
At first respiratory symptoms & $53.5 \pm 16.7$ \\
At follow-up & \\
\hline & \\
& \\
\hline
\end{tabular}

Data are presented as mean \pm SD or $\mathrm{n}(\%)$, unless otherwise indicated. Functional data were available at baseline and at the last follow-up in $89 \%$ of cases. NSIP: nonspecific interstitial pneumonia; FVC: forced vital capacity; \% pred: \% predicted; FEV1: forced expiratory volume in $1 \mathrm{~s} ; \mathrm{DL}, \mathrm{CO}$ : diffusing capacity of the lung for carbon monoxide.

though patients who later developed autoimmune diseases had a tendency to have lower interstitial score values as compared with the other patients. No differences were found in terms of pathological patterns.

\section{Pathological and radiological findings}

Among the 37 iNSIP cases initially selected from the database, the histopathology of 30 patients was reviewed. In two cases, the histological pattern was finally defined as unusual interstitial pneumonia in both lobes, and in one case a hypersensitivity pneumonitis pattern was present in one lobe (these three patients were excluded from the final analysis at the multidisciplinary approach; see fig. 1). As shown in table 1, the selected 27 iNSIP showed a "fibrosing NSIP" pattern in 15 cases and a "cellular NSIP" pattern in nine cases, whereas three cases were defined as "fibrosing NSIP plus other patterns", as the fibrosing NSIP pattern was the prevalent histopathological pattern of the specimen [22]; however, in these cases, it was also associated with areas of an organising pneumonia pattern with few fibroblastic foci in one case (patient 3; table 2), and with peribronchiolar metaplasia, honeycombing and follicular bronchiolitis in two cases (patients 13 and 18; table 2). The kappa coefficient of agreement (Cohen's kappa) between the two histopathologists who reviewed the histology was 0.61 .

The radiological analysis at baseline and at follow-up was available in 21 out of the total 27 cases. Descriptive and score features of chest HRCT scans are summarised in table 4. Among patients who developed autoimmune diseases and patients who did not, statistically significant differences were found between evaluation neither at first respiratory symptoms nor at the last follow-up; no statistically significant differences were found between patients who developed autoimmune diseases and patients who did not (either at first evaluation or at follow-up).

\section{DISCUSSION}

In the present study, we hypothesised that the clinically distinct entity iNSIP might be associated with autoimmune diseases. Our findings showed that in a patient cohort of iNSIP systematically investigated for the development of autoimmune disorders, following a multidisciplinary approach: 1) $>50 \%$ later develop an autoimmune disease (autoimmune thyroiditis, UCTD and CTD), after a mean follow-up of 22 months; 2) autoimmune thyroiditis is the most frequent autoimmune disease (26\% of cases); and 3) patients developing autoimmune diseases are older and more often never-smoking females.

It is estimated that up to $15-20 \%$ of patients who present with idiopathic ILDs either have an occult CTD or subsequently develop a CTD [23]. Thus, occult CTD might mimic idiopathic interstitial pneumonias, as the lung manifestations may often dominate the clinical picture or precede systemic findings [24]. However, these previously published data deal with idiopathic ILDs in general, and evidence suggesting an autoimmune involvement in "idiopathic" NSIP is limited.

Data on autoimmunity in iNSIP have been presented in three previous published studies [7, 8, 25]. A small series of six patients with histologically and clinically proven iNSIP, who later developed typical collagen vascular disorders was published in 2006 by a Japanese group of authors [25]. Recently, in a retrospective analysis and while assessing the clinical course and lung function change of iNSIP in an Asiatic population, PARK et al. [8] found that $10 \%$ of cases developed clinical manifestations of CTDs during follow-up. The study published by KINDER et al. [7] found that the majority of patients $(88 \%)$ classified as iNSIP met the criteria for UCTD at first lung manifestation. However, the characteristics of these three studies were significantly different from the ones in our study as, first, they were not specifically designed to carefully look for development of autoimmune diseases in a long followup of iNSIP, and, secondly, the diagnosis of iNSIP was not based on a multidisciplinary approach [5]. The study by SATO et al. [25] was a retrospective analysis including 26 patients with secondary NSIP, with underlying diseases at the time of 


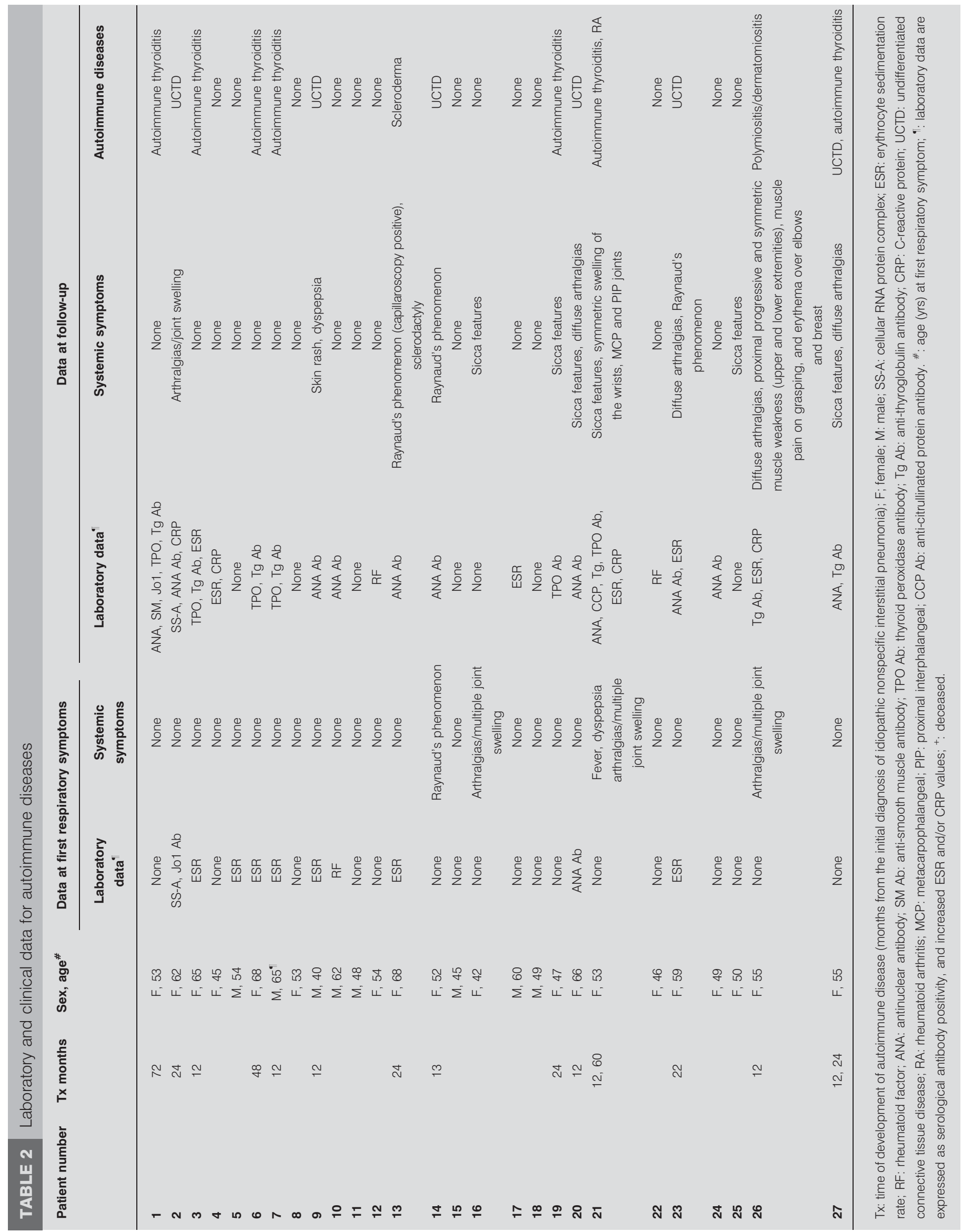


TABLE 3 Characteristics of patients who developed autoimmune diseases compared with those who did not

Patients who developed autoimmune diseases

Patients who did not develop autoimmune diseases

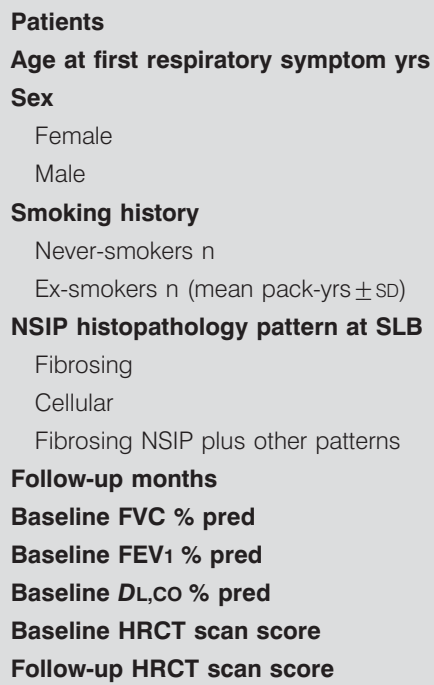

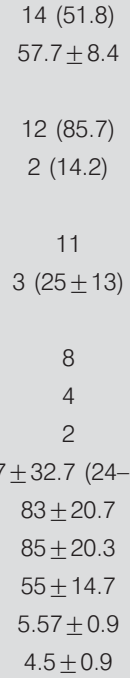

$14(51.8)$

$57.7 \pm 8.4$

$12(85.7)$

$2(14.2)$

$4.5 \pm 0.9$

8
4
2
$64.7 \pm 32.7(24-138)$
$83 \pm 20.7$
$85 \pm 20.3$
$55 \pm 14.7$
$5.57 \pm 0.9$
$4.5 \pm 0.9$

\author{
$13(48.2)$ \\ $50.5 \pm 5.9^{\#}$ \\ 7 (53.8) \\ 6 (46) \\ $4^{\#}$ \\ $9(21.4 \pm 26.7)^{\star *}$
}

$$
\begin{gathered}
7 \\
5 \\
1 \\
54.4 \pm 25.2(12-96) \\
74 \pm 18 \\
75 \pm 16 \\
61 \pm 13.7 \\
6.6 \pm 1.3 \\
5.5 \pm 0.1
\end{gathered}
$$

Data are presented as $\mathrm{n}(\%)$ and mean \pm SD, unless otherwise indicated. Unpaired t-test, Chi-squared or Fisher's exact test was used where appropriate. NSIP nonspecific interstitial pneumonia; SLB: surgical lung biopsy; FVC: forced vital capacity; \% pred: \% predicted; FEV1: forced expiratory volume in $1 \mathrm{~s}$; $D L, C O$ : diffusion capacity of the lung for carbon monoxide. HRCT: high-resolution computed tomography. $: p=0.02 ;{ }^{* *}: p=0.01$.

the histological diagnosis including CTD. In six out of the 26 patients studied, NSIP preceded $>6$ months from the onset of CTD. However, neither a description of the methods used nor the results demonstrating the manifestation of CTD were presented in that paper [25]. The aim of our study was completely different to that of the American study [7], as we deliberately excluded patients with clinical and serological manifestations of CTD and autoimmune thyroiditis at first lung manifestation. Thus, the aim of our study was to assess whether patients diagnosed with iNSIP might develop an autoimmune disease at a later time. The development of autoimmune disease, e.g. CTDs, found by PARK et al. [8] was a coincidental observation in the retrospective analysis of the clinical course and lung function change of iNSIP. In fact, this study [8] was not intended to evaluate autoimmunity in this iNSIP population. Moreover, these patients belonged to an Asiatic population, unlike the Caucasian population of our study, and no information is available concerning the likely ethnic differences in autoimmunity in iNSIP. Despite the differences between the methodology

\begin{tabular}{|c|c|c|c|c|}
\hline & $\begin{array}{l}\text { Patients who developed } \\
\text { autoimmune diseases }\end{array}$ & $\begin{array}{l}\text { Patients without } \\
\text { autoimmune diseases }\end{array}$ & $\begin{array}{l}\text { Patients with } \\
\text { autoimmune diseases }\end{array}$ & $\begin{array}{l}\text { Patients without } \\
\text { autoimmune diseases }\end{array}$ \\
\hline Patients & 12 & 9 & 12 & 9 \\
\hline Ground glass & $9(75)$ & $9(100)$ & $9(75)$ & $9(100)$ \\
\hline Interstitial score & $1.5 \pm 0.8(0-3)$ & $1.1 \pm 1(0-3)$ & $1.2 \pm 0.9(0-3)$ & $0.6 \pm 1(0-3)$ \\
\hline Traction bronchiectasis score & $1,6 \pm 1(0-3)$ & $1.1 \pm 0.9(0-2)$ & $1.16 \pm 0.7(0-2)$ & $0.7 \pm 0.8(0-2)$ \\
\hline Coarseness score & $5.5 \pm 0.9(0-12)$ & $6.6 \pm 1.3(0-14)$ & $4.5 \pm 0.9(0-14)$ & $5.5 \pm 0.1(0-14)$ \\
\hline
\end{tabular}
of the aforementioned papers $[7,8,25]$ and our present study, all these findings together seem to support the hypothesis that an autoimmune background might play an important role in the pathogenesis of iNSIP, and that iNSIP might represent the early lung manifestation of an autoimmune disease, as previously hypothesised [5], although the precise pathogenetic mechanisms are still unknown.

TABLE 4 Chest high-resolution computed tomography (HRCT) scan features

Data are presented as $\mathrm{n}$ or mean $\pm \mathrm{SD}$ (range). 
The association between CTD and autoimmune thyroid disease is well recognised. The most frequent one is the association of rheumatoid arthritis, Sjögren's syndrome and autoimmune thyroiditis [26, 27], and the incidence of CTD seems to be higher in patients suffering from autoimmune thyroiditis [28]. The specific rationale to investigate thyroid autoimmune disorders in our iNSIP patients was based on the hypothesis of its likely link with thyroid disorders, since both lung and thyroid share the thyroid transcription factor-1, which plays a role in the development and physiology of both thyroid and lungs. Our data on the development of autoimmune thyroiditis in iNSIP support single case reports describing an association of NSIP and chronic/autoimmune thyroiditis $[29,30]$. The histopathological lesions of the thyroid gland in autoimmune thyroiditis (organ-specific autoimmune disease), and of salivary and lachrymal glands in Sjögren's syndrome are quite similar, characteristically showing focal or diffuse T-lymphocytic infiltrates [31,32], suggesting that both diseases share pathogenetic pathways. Intriguingly, NSIP also typically shows T-lymphocytic infiltrates in the lung interstitium, along with focal B-cell accumulation and fibrosis [1]. Based on these observations, we would speculate that "iNSIP" might be a so far unrecognised organ-specific autoimmune disorder, such as an autoimmune pneumonitis, rather than a latent autoimmune disorder [7]. However, we are aware that there is, at present, no real evidence to either prove or exclude this hypothesis.

The iNSIP population of the present analysis showed clinical similarities to the study populations more recently described $[5,8]$, with a predominance of never-smoking, middle-aged females, with substantial stable lung function at follow-up [4, 33]. Compared with the study by PARK et al. [8], we found a higher incidence of the development of CTD during follow-up (a total of 33\% in our analysis, including CTD and UCTD, as compared with $10 \%$ in their study, including CTD and mixed CTD), maybe as a result of our systematic approach for detection and/or due to possible ethnic differences in developing autoimmune disorders. Moreover, the most important difference between our autoimmune results and those of PARK et al. [8] was the high incidence of autoimmune thyroiditis we found $(26 \%$ in total, representing almost the half of the total autoimmune diseases), which was neither considered nor investigated in the previous study [8].

The present study has several limitations, as follows: it is a descriptive study, only speculating on likely pathogenetic mechanisms and including a relatively small number of patients. However, although the population of the present analysis is relatively small, it was carefully characterised at a multidisciplinary approach, and it must be highlighted that iNSIP is an uncommon disease, and it is therefore quite difficult to collect large numbers of patients. The present analysis was made only in patients with complete autoimmune information (seven patients were excluded because of incomplete data), which might lead to the "complete case" analysis bias, as previously highlighted [34]. However, even if these excluded patients had not developed any autoimmune disorder, the percentage of cases developing autoimmune diseases would still have been more than one-third of the total study population. Conversely, considering that the mean interval of time to develop an autoimmune disease was
22 months, with a range from 12 to 72 months from the initial diagnosis of iNSIP, some of our included patients potentially might still develop an autoimmune disease in the future.

Despite these limitations, the present study is the only analysis to date presenting carefully and systematically collected serological and clinical data of autoimmune diseases during a long-term follow-up of patients with iNSIP, characterised by surgical lung biopsies, and with a final diagnosis based on a multidisciplinary approach. The results of this study emphasise the need for a complete autoimmune assessment in the follow-up of patients with iNSIP, including thyroid disorders. Thus, the development of autoimmune thyroiditis in the $26 \%$ of cases of this study population represents a new finding. To the best of our knowledge, no previously published paper on iNSIP has ever shown this finding. The data related to the group of patients who did not develop any autoimmune disease (younger, predominantly smoking) might support the hypothesis of another NSIP phenotype, with a possible smoking pathogenesis, as previously suggested $[9,10]$.

In conclusion, we present here the largest patient cohort of iNSIP so far systematically investigated for the development of autoimmune disorders. The study highlights the high prevalence of a manifestation of autoimmunity in patients initially diagnosed with "idiopathic" NSIP, and adds a striking incidence of autoimmune thyroiditis to the existing literature, indicating that a long-term follow-up with a multidisciplinary clinical approach and evaluation for autoimmune diseases is mandatory in these patients. Although, due to the limited data, a definite conclusion cannot be drawn, this study suggests a probable link between the clinical entity of iNSIP and autoimmune disorders. Future studies should confirm these findings.

\section{STATEMENT OF INTEREST}

None declared.

\section{ACKNOWLEDGEMENTS}

The authors gratefully acknowledge M. Buccioli for his helpful assistance as data manager of our pulmonology unit.

\section{REFERENCES}

1 Katzenstein AL, Fiorelli RF. Nonspecific interstitial pneumonia/ fibrosis. Histologic features and clinical significance. Am J Surg Pathol 1994; 18: 136-147.

2 Flaherty KR, Martinez FJ. Nonspecific interstitial pneumonia. Semin Respir Crit Care Med 2006; 27: 652-658.

3 American Thoracic Society, European Respiratory Society. American Thoracic Society/European Respiratory Society International Multidisciplinary Consensus Classification of the Idiopathic Interstitial Pneumonias. This joint statement of the American Thoracic Society (ATS), and the European Respiratory Society (ERS) was adopted by the ATS board of directors, June 2001 and by the ERS Executive Committee, June 2001. Am J Respir Crit Care Med 2002; 165: 277-304.

4 Martinez FJ. Idiopathic interstitial pneumonias: usual interstitial pneumonia versus nonspecific interstitial pneumonia. Proc Am Thorac Soc 2006; 3: 81-95.

5 Travis WD, Hunninghake G, King TE Jr, et al. Idiopathic nonspecific interstitial pneumonia: report of an American Thoracic Society project. Am J Respir Crit Care Med 2008; 177: 1338-1347. 
6 Romagnoli M, Gurioli C, Casoni G, et al. Surgical lung biopsy in the diagnosis of idiopathic NSIP: do we always need it in the initial approach? Am J Respir Crit Care Med 2009; 179: 1071.

7 Kinder BW, Collard HR, Koth L, et al. Idiopathic nonspecific interstitial pneumonia: lung manifestation of undifferentiated connective tissue disease? Am J Respir Crit Care Med 2007; 176: 691-697.

8 Park IN, Jegal Y, Kim DS, et al. Clinical course and lung function change of idiopathic nonspecific interstitial pneumonia. Eur Respir J 2009; 33: 68-76.

9 Marten K, Milne D, Antoniou KM, et al. Non-specific interstitial pneumonia in cigarette smokers: a CT study. Eur Radiol 2009; 19: 1679-1685.

10 Craig PJ, Wells AU, Doffman S, et al. Desquamative interstitial pneumonia, respiratory bronchiolitis and their relationship to smoking. Histopathology 2004; 45: 275-282.

11 Hochberg MC. Updating the American College of Rheumatology revised criteria for the classification of systemic lupus erythematosus. Arthritis Rheum 1997; 40: 1725.

12 Lonzetti LS, Joyal F, Raynauld JP, et al. Updating the American College of Rheumatology preliminary classification criteria for systemic sclerosis: addition of severe nailfold capillaroscopy abnormalities markedly increases the sensitivity for limited scleroderma. Arthritis Rheum 2001; 44: 735-736.

13 Tanimoto K, Nakano K, Kano S, et al. Classification criteria for polymyositis and dermatomyositis. J Rheumatol 1995; 22: 668-674.

14 Vitali C, Bombardieri S, Jonsson R, et al. Classification criteria for Sjögren's syndrome: a revised version of the European criteria proposed by the American-European Consensus Group. Ann Rheum Dis 2002; 61: 554-558.

15 Venables PJ. Mixed connective tissue disease. Lupus 2006; 15: 132-137.

16 Doria A, Mosca M, Gambari PF, et al. Defining unclassifiable connective tissue diseases: incomplete, undifferentiated, or both? J Rheumatol 2005; 32: 213-215.

17 Mosca M, Neri R, Bombardieri S. Undifferentiated connective tissue diseases (UCTD): a review of the literature and a proposal for preliminary classification criteria. Clin Exp Rheumatol 1999; 17: 615-620.

18 Dayan CM, Daniels GH. Chronic autoimmune thyroiditis. N Engl J Med 1996; 335: 99-107.

19 Pedersen OM, Aardal NP, Larssen TB, et al. The value of ultrasonography in predicting autoimmune thyroid disease. Thyroid 2000; 10: 251-259.
20 Hansell DM, Bankier AA, MacMahon H, et al. Fleischner Society: glossary of terms for thoracic imaging. Radiology 2008; 246: 697-722.

21 Akira $M$, Inoue $Y$, Kitaichi $M$, et al. Usual interstitial pneumonia and nonspecific interstitial pneumonia with and without concurrent emphysema: thin-section CT findings. Radiology 2009; 251: 271-279.

22 Katzenstein A. Idiopathic interstitial pneumonia: classification and diagnosis. In: Katzenstein A, Askin FB, eds. Surgical pathology of non-neoplastic lung disease. Philadelphia, W B Saunders, 1997; pp. 1-31.

23 Strange $\mathrm{C}$, Highland KB. Interstitial lung disease in the patient who has connective tissue disease. Clin Chest Med 2004; 25: 549-559, vii.

24 Tzelepis GE, Toya SP, Moutsopoulos HM. Occult connective tissue diseases mimicking idiopathic interstitial pneumonias. Eur Respir J 2008; 31: 11-20.

25 Sato T, Fujita J, Yamadori I, et al. Non-specific interstitial pneumonia; as the first clinical presentation of various collagen vascular disorders. Rheumatol Int 2006; 26: 551-555.

26 Perez B, Kraus A, Lopez G, et al. Autoimmune thyroid disease in primary Sjogren's syndrome. Am J Med 1995; 99: 480-484.

27 Pyne D, Isenberg DA. Autoimmune thyroid disease in systemic lupus erythematosus. Ann Rheum Dis 2002; 61: 70-72.

28 Soy M, Guldiken S, Arikan E, et al. Frequency of rheumatic diseases in patients with autoimmune thyroid disease. Rheumatol Int 2007; 27: 575-577.

29 Hashizume T, Numata H, Matsushita K. Interstitial pneumonia associated with chronic thyroiditis. Nihon Kokyuki Gakkai Zasshi 2002; 40: 31-34.

30 Khardori R, Eagleton LE, Soler NG, et al. Lymphocytic interstitial pneumonitis in autoimmune thyroid disease. Am J Med 1991; 90: 649-652.

31 Adamson TC 3rd, Fox RI, Frisman DM, et al. Immunohistologic analysis of lymphoid infiltrates in primary Sjogren's syndrome using monoclonal antibodies. J Immunol 1983; 130: 203-208.

32 Mason DK, Harden RM, Alexander WD. The salivary and thyroid glands. A comparative study in man. Br Dent J 1967; 122: 485-489.

33 Flaherty KR, Thwaite EL, Kazerooni EA, et al. Radiological versus histological diagnosis in UIP and NSIP: survival implications. Thorax 2003; 58: 143-148.

34 Chen H, Gomez A, Shum A, et al. Longitudinal lung function in idiopathic NSIP: what are we missing? Eur Respir J 2009; 34: 1004. 